\title{
Estrategias de movilidad, visados y fronteras: Trayectorias de haitianos y haitianas hacia la Argentina
}

\section{Mobility strategies, visas and borders: Trajectories of Haitians towards Argentina}

Carina Trabalón * (iD https://orcid.org/0000-0003-3675-2338
Recibido el 6 de septiembre de 2019 Aceptado el 16 de diciembre de 2019. Publicado el 20 de diciembre de 2019.

*Autor para correspondencia: Carina Trabalón, correo electrónico, carinatrabalon522@gmail.com

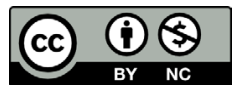

Esta obra está protegida bajo una Licencia Creative Commons Atribución-NoComercial 4.0 Internacional.

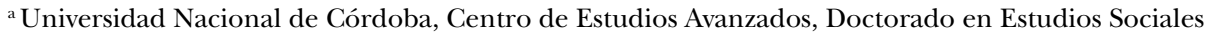
de América Latina, Argentina, correo electrónico: carinatrabalon522@gmail.com
\end{abstract}

\section{Resumen:}

En el presente artículo se abordan diferentes aspectos referidos a la configuración de las fronteras y el desarrollo de las estrategias de movilidad de haitianos y haitianas que residen en Argentina, desde mediados del 2000 al presente. Las fricciones entre los "destinos ideales" y los "destinos posibles", los distintos modos en que Argentina se incorpora en la definición de los proyectos migratorios, y las transformaciones que se producen entre las experiencias de cruce de frontera y las regulaciones estatales de control, son algunas de las dimensiones que permiten poner de relieve las tensiones entre movilidad, nacionalidad, racialización y fronteras. Así, desde un enfoque metodológico cualitativo, basado en observación participante y entrevistas en profundidad a haitianos y haitianas en las ciudades de Córdoba y Rosario, se analizan los vínculos entre las estrategias migrantes, sus trayectorias a lo largo del tiempo y la regulación estatal a través de los visados y controles fronterizos. La consideración de estas trayectorias en diversos momentos y espacios muestra cómo las políticas de visados no solo implican claras restricciones a la movilidad sino que, además, su ausencia no se traduce necesariamente en posibilidades de admisión irrestricta a los territorios extranjeros.

Palabras clave: fronteras, políticas de visado, control, migración haitiana, trayectorias, procesos de racialización.

\section{Abstract:}

This article aims to address different aspects related to the configuration of borders and the development of Haitian mobility strategies that reside in Argentina, from mid-2000 to the present. The frictions between the "ideal destinations" and the "possible destinations", the different ways in which Argentina is incorporated into the definition of migratory projects, and the transformations that

CÓMO CITAR: Trabalón, C. (2019). Estrategias de movilidad, visados y fronteras: Trayectorias de haitianos y haitianas hacia la Argentina. [Mobility strategies, visas and borders: Trajectories of Haitians towards Argentina]. Estudios Fronterizos, 20 , e039. doi:https://doi.org/10.21670/ref.1918039 
occur between border crossing experiences and state control regulations, are some of the dimensions that allow highlighting the tensions between mobility, nationality, racialization and borders. Thus, from a qualitative methodological approach, based on participant observation and in-depth interviews with male and female Haitians in the cities of Córdoba and Rosario, the links between migrant strategies, their trajectories over time and state regulation are analyzed through of visas and border controls. The consideration of these trajectories at various times and spaces shows how visa policies not only imply clear restrictions on mobility but also that their absence does not necessarily translate into unrestricted admission to foreign territories.

Keywords: borders, visa policies, control, Haitian migration, trajectories, racialization processes.

\section{Introducción}

Durante las últimas décadas, el desarrollo del capitalismo global ha exhibido de manera cada vez más evidente la conformación de un sistema diferencial y jerárquico a través del cual las desigualdades sociales son reforzadas en tanto son reproducidas como desigualdades de acceso a la movilidad (Neumayer, 2006). Así, en tiempos de globalización, los Estados intentan conciliar los beneficios económicos y políticos que trae aparejada la movilidad global con la prerrogativa que poseen para controlar sus fronteras (Sassen, 2007). En este escenario, emergen y se agudizan las tensiones entre movilidades, fronteras y nacionalidad, así como también sobresalen los distintos marcadores — de clase, raza, género, etnia, edad, religión, entre otros- que intervienen en la estructuración de las movilidades (Glick-Schiller y Salazar, 2013). De este modo, las distintas nacionalidades se insertan en un sistema de poder desigual en el que determinados ciudadanos y sus pasaportes poseen más valor que otros (Balibar, 2012). Como correlato, no sorprende constatar que los grupos nacionales con menos posibilidades de movilidad a escala global, es decir, sin mediación de visados consulares, correspondan a los nacionales de Haití (cuyo pasaporte tiene la mayor cantidad de restricciones a nivel latinoamericano), Cuba, la mayoría de los países africanos y algunos de Asia y Medio Oriente ("Estos son los pasaportes", 2019; McKirdy, 2019; Yu, 2016).

En este sentido, se destacan las profundas transformaciones que tienen lugar desde mediados de los noventa en el campo de la política internacional y, como resultado, en las diferentes formas de comprender la soberanía (Mezzadra, 2012). Así, en términos generales, mientras que la noción de "régimen global de control de las migraciones" (Düvell, 2003) permite dar cuenta de mecanismos, discursos y prácticas específicas a través de los cuales las "técnicas administrativas de control, las 'normas' técnicas y los programas de capacitación circulan a escala global, ejerciendo gran influencia en la formulación de políticas migratorias nacionales" (Mezzadra, 2012, p. 169), el proceso de regionalización de la política migratoria refiere, entre otras cuestiones, a la penetración/adaptación de diferentes aspectos del modelo de gobernabilidad migratoria que se viene observando en las últimas dos décadas en América Latina, junto con el surgimiento de nuevas formas de clasificar y organizar a la migración y a los migrantes (Domenech, 2007, 2013, 2017). En esta línea, se propone conectar las experiencias de jóvenes haitianos hacia la Argentina con la reconfiguración de 
las políticas de control migratorio y fronterizo, y el aumento de las restricciones de visados a partir de diferentes escalas, espacios y temporalidades. ${ }^{1}$

Estudios producidos en otros contextos, como Estados Unidos, Canadá y Europa, han definido las visas consulares como una forma de particular control fronterizo que implica la preselección de potenciales viajeros para la admisión a los distintos territorios nacionales (Guild y Bigo, 2003; Neumayer, 2006; Salter, 2006; Salter y Mutlu, 2010; Zolberg, 2003). Así, la deslocalización de la función de la frontera permitiría a los Estados participar de la clasificación del comportamiento de los viajeros fuera de sus límites físicos. En esta línea, podría decirse que limitar la entrada al territorio es la manera más efectiva que tienen los Estados para evitar acceder a una serie de obligaciones con los no nacionales considerados "indeseables" y, por ello, los controles de pasaportes y visados pasan a constituirse como la "primera línea de defensa" para el control de los ingresos y admisiones (Torpey, 1998). Por otro lado, la significación de los visados en espacio sudamericano, si bien ha sido destacada por algunos autores (Domenech, 2017; Salmón, 2017), se observa cierta vacancia en relación con el estudio sistemático de estas políticas. A pesar de ello, se recuperan diversas investigaciones pertenecientes al campo de estudios sobre políticas migratorias que permiten abordar y pensar el tratamiento de esta temática desde un análisis situado socio-históricamente.

Asimismo, dentro del campo de los estudios fronterizos se ha señalado que muchos trabajos restringen el análisis de las fronteras al quehacer de actores estatales y paraestatales (Casa-Cortes, Cobarrubias, De Genova et al, 2015). En este sentido, se propone un enfoque constructivista de la frontera que permita entenderla como un sitio de constante encuentro, conflicto y negociación y, en consecuencia, abordar su producción, no solo desde las lógicas gubernamentales, sino también desde los propios movimientos de la migración (Casa-Cortes, Cobarrubias, De Genova et al., 2015; Casas-Cortes, Cobarrubias y Pickles, 2015; Mezzadra y Neilson, 2017; Papadopoulos y Tsianos, 2013). Desde esta perspectiva, la noción de régimen fronterizo habilita a pensar el conjunto heterogéneo de actores, prácticas y discursos que convergen, no sin contradicciones, en aquellos procesos históricamente situados que configuran las fronteras, permitiendo pasar del análisis de la frontera al análisis de sus procesos de constitución. En esta línea, la frontera como institución social y conjunto de relaciones sociales se aleja de aquellas propuestas que la definen en términos exclusivamente territoriales y/o geopolíticos y postula, en cambio, un abordaje a partir de la proliferación y heterogeneización de las mismas (Mezzadra y Neilson, 2017).

En este punto cobran especial relevancia los procesos de racialización presentes en la configuración de los esquemas de control migratorio y fronterizo. En este sentido, se recupera la noción de "colonialidad del poder" de Quijano, referida a la constitución del patrón mundial capitalista que está fundado en:

[...] la imposición de una clasificación racial/étnica de la población del mundo como piedra angular de dicho patrón de poder y opera en cada uno de los planos, ámbitos y dimensiones, materiales y subjetivas, de la existencia social cotidiana y a escala societal (Quijano, 2000, p. 342).

\footnotetext{
${ }^{1}$ En este sentido, el análisis también se orienta a partir de una propuesta que busca conectar las experiencias de los sujetos con procesos más generales de estructuración de las desigualdades, teniendo en cuenta para ello diferentes escalas, espacios y temporalidades (Feldman-Bianco, 2015; 2018).
} 
Al mismo tiempo, como argumenta Segato (2011), la raza como categoría social construida históricamente responde en cada país a formaciones nacionales de la alteridad, de manera que, la construcción de la nación supone en cada caso una determinada producción de "la raza como marca de exterioridad en relación con los centros de poder nacional" (Segato, 2011, p. 7). En el caso argentino, la formación de la nación supuso la puesta en práctica de diferentes mecanismos tendientes a la homogeneización étnica mediante procesos de blanqueamiento específicos. Así, la referencia al "crisol de razas" en Argentina remite exclusivamente a grupos nacionales europeos, al tiempo que, niega e invisibiliza toda presencia de la alteridad indígena y afrodescendiente del proyecto nacional (Segato, 2007).

En este marco, tomando como punto de partida las prácticas de los sujetos entendidas en términos de estrategias, es decir, sus disposiciones a actuar, pensar y orientarse de acuerdo con la posición social ocupada y un sentido práctico del juego social (Bourdieu, 1997), interesa comprender sus desarrollos en conexión con la constitución de las fronteras. En esta dirección, se privilegia el análisis de aquellas estrategias que, desplegadas en contextos de movilidad, permiten dar cuenta de las tensiones y reconfiguraciones que se producen entre las prácticas de atravesamiento y las prácticas de reforzamiento fronterizo (Vila, 2000), prestando especial atención a los efectos de las categorías nacionales (Sayad, 2008) y prácticas estatales que intervienen en las lógicas de control. De acuerdo con esto, se sostiene que el visado de turismo impuesto en Argentina en agosto de 2018 para nacionales haitianos se constituye en un contexto de producción de políticas más amplio en el que confluyen diversos actores, prácticas y discursos. Sin perder de vista esta confluencia, el presente artículo se circunscribe al análisis de las experiencias de jóvenes haitianos y haitianas que residen en las ciudades de Córdoba y Rosario, por un lado, a partir de la construcción de sus proyectos migratorios en origen y la conformación de estrategias de movilidad hacia la Argentina y, por otro, en relación con los cambios que se observan en las percepciones sobre el control migratorio y fronterizo y el desarrollo de las estrategias de cruce de frontera a lo largo de las distintas trayectorias.

En este sentido, desde un enfoque cualitativo, basado en la observación participante en diferentes espacios sociales y en entrevistas en profundidad a jóvenes haitianos y haitianas que residen en las ciudades de Córdoba y Rosario, a lo largo del artículo se analizarán fragmentos de entrevistas y registros de campo que remiten a distintas experiencias, con el objetivo de resaltar ciertos ejes transversales, pero también algunas diferencias que dan cuenta de la heterogeneidad presente en las distintas trayectorias. ${ }^{2}$ De este modo, el abordaje de las experiencias en relación con las políticas de visados pretende contribuir al debate sobre las formas que adquieren las relaciones entre Estado, nacionalidad y fronteras en el marco de las movilidades sursur y las reconfiguraciones que se observan en las últimas dos décadas en Argentina y la región. Para ello, se analizan prácticas concretas que dan cuenta del carácter selectivo y la función de filtrado que tienen las fronteras, pero también de sus porosidades y posibilidades de atravesamiento. Con el énfasis puesto en las trayectorias de los sujetos, se intenta mostrar que la existencia de visados consulares, no solo implica claras restricciones a la movilidad, sino que, además, su ausencia tampoco se traduce necesariamente en posibilidades de admisión irrestricta al territorio.

${ }^{2}$ Se reservarán los nombres verdaderos y ciertos datos específicos con el objetivo de mantener el anonimato de mis interlocutores haitianos y haitianas. 
La estructura del trabajo se organiza en tres apartados. En primer lugar, se exponen algunas particularidades de las trayectorias de haitianos en las ciudades de Córdoba y Rosario para un análisis situado de las mismas. En segundo lugar, se examina el lugar que ocupan los visados en la conformación de los proyectos migratorios de jóvenes haitianos con respecto a países como Estados Unidos, Francia o Canadá en contraste con los modos en que se define Argentina como "destino" según diferentes temporalidades. Por último, se realiza una aproximación a los procesos de constitución de la política de visado en Argentina para poner en evidencia las tensiones entre estrategias de movilidad y rechazos en frontera a partir de algunos de los marcadores sociales que operan en el control fronterizo a lo largo de las distintas trayectorias.

\section{Proyectos migratorios de jóvenes haitianos y haitianas hacia la Argentina}

A continuación, interesa destacar algunos aspectos generales, pero significativos, de la movilidad de haitianos hacia las ciudades de Rosario y Córdoba para un análisis situado de sus relaciones y conexiones con el control migratorio y fronterizo. Al reinscribir sus trayectorias en el marco de diversos procesos globales y regionales se busca poner de relieve las tensiones y reconfiguraciones que se establecen entre movilidad, fronteras y nacionalidad en diferentes escalas, espacios y temporalidades. Con esta finalidad, se recuperan elementos trabajados en distintas investigaciones sobre migración haitiana en la región. ${ }^{3}$ Gran parte de estos estudios analizan la movilidad de haitianos hacia países sudamericanos como Ecuador, Perú, Brasil, Chile e, incluso Argentina, a partir del impulso que generó el terremoto ocurrido en Haití en 2010, el cual habría agudizado las ya frágiles e inestables condiciones sociales, políticas y económicas en las que se encontraba el país. Asimismo, más allá de que la migración haitiana en Sudamérica se considera reciente, suele subrayarse que se trata de una migración antigua, dirigida históricamente a múltiples destinos, entre los que sobresalen algunos países del Caribe (como República Dominicana y Cuba), Estados Unidos, Canadá y Francia. Así, la migración haitiana en general, incluidos los nuevos movimientos hacia los países sudamericanos, se asocia fundamentalmente con las condiciones estructurales que lo ubican como uno de los países más pobres a nivel regional, así como con la recurrente inestabilidad y violencia política que se observa a nivel institucional.

En el caso de las ciudades de Rosario y Córdoba, los proyectos migratorios de estos jóvenes han sido definidos principalmente a partir del interés personal y/o familiar de realizar sus estudios universitarios en Argentina. A lo largo de estos años se ha advertido cómo estas aspiraciones se han encontrado con diversos obstáculos -culturales, sociales y políticos- que han resultado, para muchos, en el abandono de sus estudios universitarios, la inserción en mercados laborales precarizados y/o la circulación hacia otras ciudades, países e, incluso, el retorno a Haití. Sin entrar en detalle sobre estas dificultades, el análisis se centra en las experiencias de aquellos

\footnotetext{
${ }^{3}$ Entre ellos: Alarcón, 2015; Berganza, 2017; Burey, 2018; Ceja, 2015; De Heusch, 2016; Duffard, 2016; Fernandes y Faria, 2017; Nieto, 2014; OBIMID, 2016; OIM-FCCAM, 2017; Pedemonte, Amode y Rencoret, 2015; Pedemonte, Amode y Vásquez, 2017; Silva, 2015; Thomaz, 2013; Véran, Da Silva y Fainstat, 2014.
} 
haitianos y haitianas que, más allá de estar trabajando o no, están vinculados aún con el proyecto educativo universitario, ya sea intentando ingresar a la universidad, realizando actualmente sus estudios en distintas carreras o, también, habiendo finalizado sus estudios universitarios, insertándose profesionalmente en distintas áreas.

En cuanto a la conformación de sus proyectos migratorios, en lo que sigue se analizan los casos de tres jóvenes por considerar que expresan de manera paradigmática algunas de las características que asumen y atraviesan gran parte de las diferentes experiencias conocidas. En particular, aquellas referidas al carácter familiar y transnacional de los proyectos migratorios y las trayectorias sociales y generacionales con base en las cuales se generan las condiciones para llevar adelante dichos proyectos.

El primer caso es el de Robert, uno de los muchos haitianos que llegaron entre mediados y fines del 2000. Él cuenta que sus padres, ambos comerciantes, no tuvieron la posibilidad de acceder a los estudios, pero que trabajaron toda su vida en Port-dePaix, región noroeste de Haití, con el objetivo de que él y sus hermanos pudieran acceder a los estudios universitarios y convertirse en profesionales. Este joven de 29 años, que llegó a la Argentina en 2008, participa en una asociación de Rosario y es un referente para muchos jóvenes que residen en la ciudad, quienes acuden a él para consultar sobre diferentes temáticas. Referido en particular a la definición de los proyectos migratorios de los jóvenes haitianos que llegan a esta ciudad, en el relato de Robert se advierte un estrecho vínculo entre la familia, la educación y el carácter trasnacional de los mismos. Según sus palabras:

[...] la familia en Haití dice bueno yo pago la facultad te pago la escuela, vos tenés que estudiar, yo trabajo, no te preocupes, yo trabajo vos tenés que estudiar, lo único que te pido es estudiar, estudiá. Es así las familias en Haití, cuando uno llega, eso es muy importante, cuando un haitiano llega, llega una familia, llega un barrio, toda una zona, el tío, la tía, estando acá, el vecino, la vecina, el tío, la tía, el papá, la mamá, los hermanos, todos te llaman, ¿cómo estás? ¿Cómo va el estudio? Porque tienen fe, y están esperando una buena noticia, cuando llegás se siente "yo tengo mi hijo que es médico", "mi vecino es médico, estudió en Argentina", "mi primo es médico", toda la gloria es para ellos. Cuando uno fracasa acá hay una banda de familias que fracasan con uno, cuando uno llega, llega una banda de familias (Robert, 16 de noviembre, 2017).

En segundo lugar, Joseph es un joven haitiano de 31 años que vivió toda su vida en Port-au-Prince, capital de Haití, y llegó a la ciudad de Rosario en 2011. Si bien Joseph no forma parte de ninguna asociación, promueve y participa activamente en diferentes actividades que tienen por objetivo presentar a su país y su cultura, pero también reflexionar sobre su situación y condición como migrante/s haitiano/s en Argentina. Al igual que Robert, Joseph comenta acerca del esfuerzo y el significado que tenía para sus padres el hecho de que él y sus hermanos pudieran acceder a la educación: "ellos no sabían leer [...] Existe eso de los padres, porque hay mucha gente allá que no sabe leer. Ellos dicen 'yo no tuve esa oportunidad' o 'esa suerte' como dicen allá; 'a mi hijo eso no le va a pasar'. Entonces hacen mucho esfuerzo” (Joseph, 17 de noviembre, 2017). 
En tercer lugar, se recupera el caso de Moise, un joven de 22 años que llegó a Córdoba en 2016 desde Les Cayes, región sur de Haití. Moise también es un referente para los haitianos que viven en Córdoba y forma parte de una de las tres asociaciones que existen en esta ciudad, donde el número de haitianos residentes es bastante menor al de Rosario. Si bien Moise cuenta que sus padres pudieron terminar sus estudios secundarios y su madre estudiar magisterio, también comparte la mirada de Robert y Joseph sobre la importancia asignada por los padres a la educación:

El padre haitiano... Hay algo en su cabeza que, "no me importa que voy caminando descalzo para pagar el estudio de mis hijos. Es lo que más me importa”. Lo que más le importa es el estudio de su hijo y andan descalzos si es necesario. [...] Porque en Haití hay un dicho que dice que el hijo es la riqueza de los padres, digamos [...] trabaja para que sus hijos puedan llegar a un lugar más alto, lo más alto que puedan [...] porque saben que algún día ese hijo, si tiene buena fe, si tiene buen corazón, le va a dar una mano y lo va a sacar de la situación donde estaba. Eso pasó con muchas familias haitianas que los padres hicieron un esfuerzo enorme y bueno, llegando a un cierto nivel, entonces los hijos al nivel que llegaron les empezaron a ofrecer a los padres viajes...Digamos, ofrecerles una vida mejor (Moise, 6 de abril, 2018).

En estos tres casos, así como en la mayoría de los relatos de los jóvenes, se muestra una idea compartida en relación con las dificultades de ingresar a las universidades públicas, el costo de las universidades privadas y la mayor valoración que tendrían los títulos obtenidos en el extranjero en contraste con aquellos conseguidos en las universidades de Haití. Asimismo, en varias ocasiones también se vinculó el hecho de estudiar fuera de país con una experiencia usual que los jóvenes con aspiraciones profesionales realizan una vez terminados sus estudios secundarios. También se resaltó la importancia de tener familiares en otros países como Estados Unidos, Canadá o Francia que aporten económicamente a sus proyectos en los primeros meses o a los que se puede recurrir, eventualmente, para solicitar la ayuda económica necesaria para darle continuidad a sus estudios. De acuerdo con esto, podría decirse que las trayectorias de haitianos y haitianas hacia las ciudades de Rosario y Córdoba están ligadas a un proyecto familiar en el que la educación aparece en la mayoría de los casos como estrategia de movilidad social construida generacionalmente y, por lo general, vinculada a trayectorias migrantes familiares de distintos tipos. En este sentido, se considera al proyecto migratorio como parte de las estrategias de reproducción social de la familia, entendidas, más que como intenciones conscientes y a largo plazo, como el conjunto de acciones tendientes a aumentar o a conservar su patrimonio, manteniendo o mejorando su posición en la estructura de las posiciones de clase (Bourdieu, 2011). A su vez, de este rasgo familiar también se desprende el carácter transnacional de los proyectos y la conexión entre diferentes lugares y prácticas. Es decir, la referencia de uno de los entrevistados de que "cuando un haitiano llega, llega toda la familia" remite precisamente a la formación de redes de relaciones sociales (Feldman-Bianco, 2015; Levitt y Glick-Schiller, 2004) que conectan, de manera simultánea, las prácticas de los jóvenes en su localidad de residencia, no solo con Haití, sino con los diferentes lugares en los que viven sus familiares y amigos. 


\section{El visado como regulador de la movilidad: Entre "destinos ideales" y ¿destinos posibles?}

Con el pasaporte haitiano no tenés tantos accesos, por ejemplo. Incluso tenía una discusión con una francesa. Yo decía, "Bueno, ahora hay más haitianos en Chile que en Francia". Y ella me dice, "No, hay otros países mejores para viajar". Dije, "bueno, vos sí con tu pasaporte podés viajar a todo el mundo", pero el haitiano no tiene tanta posibilidad. Por ejemplo, "vamos a Dinamarca", pero no, para ir a Dinamarca es como, “¿Qué va a hacer un haitiano en Dinamarca?”. Los trámites que tenés que hacer... (Joseph, 17 de noviembre, 2017).

Teniendo en cuenta las particularidades mencionadas, se aborda el lugar que ocupan las prácticas de control migratorio y fronterizo en la definición de los proyectos migratorios de los jóvenes y sus estrategias de movilidad hacia la Argentina. En esta dirección, se analizan las percepciones y prácticas en torno a las políticas de visados: primero, en conexión con los "destinos" históricamente construidos de Estados Unidos, Francia y Canadá; segundo, a partir del contraste entre estos países y los "nuevos" países sudamericanos y, en especial, sobre los diferentes modos en que Argentina se construye como "destino".

Con respecto al primer punto, en muchas de las charlas sostenidas y entrevistas realizadas, se deja entrever una diferenciación y jerarquización entre los que se consideran los países más "deseados" o "ideales" para migrar, es decir, Estados Unidos, Francia y Canadá; y, por otro lado, los "nuevos" países sudamericanos que han emergido como posibilidad en los últimos años. En este marco, las referencias a los "destinos ideales" se manifiestan en los relatos, la mayoría de las veces, como reflexiones sobre lo que se pensaba con respecto a estos países en el momento en que fueron definidos sus proyectos migratorios. Sin embargo, aparecen también como menciones - en ocasiones, críticas - a los idearios haitianos sobre la migración y a las representaciones idílicas que tendrían muchos jóvenes y sus familias sobre estos lugares. Asimismo, en varios casos, se remite a estos países como parte de los proyectos migratorios actuales, es decir, algunos jóvenes que residen en las ciudades de Córdoba y Rosario consideran estos "destinos" aún como el principal objetivo a alcanzar.

Como sostiene Michele (14 de marzo de 2018), una de las jóvenes entrevistadas, "en general, hay una tendencia a ir a Estados Unidos y Canadá, en el haitiano. ¿Por qué? Por esa cuestión, que siempre hay familiares ahí. Por un ideal que se hacen, digamos, del tipo de vida allá [...]". De este mismo ideal nos habla Moise cuando señala con respecto a sus amigos del secundario que "los que fueron al colegio, [...] uno de los mejores allá, la idea, la gente de clase media y media alta, son hijos que terminan la secundaria, se van a Francia, Canadá o Estados Unidos. Así que tengo un montón de compañeros que están en Estados Unidos, la mayoría. Los otros están en Canadá o Francia” (Morse, 6 de abril, 2018). Por su parte, Joseph comenta que estos "destinos" siguen considerándose como el "escalón más alto a alcanzar" para muchos de los jóvenes, y, al mismo tiempo, manifiesta que "seguimos pensando en eso (en viajar a Estados Unidos, Canadá o Francia). Pero cada día es más complicado [...], piden un montón de cosas..." (Joseph, 17 de noviembre, 2017). En relación con la persistencia en el deseo de viajar a estos países, según Robert "ir a Estados Unidos, 
Canadá o Francia es lo que más se buscaba, en realidad todavía muchos lo siguen pensando, están acá, pero tienen la cabeza allá" (Robert, 16 de noviembre, 2017). Precisamente lo expresado por Robert aparece ejemplificado en el caso de Pierre, quien al momento de la entrevista se encontraba realizando los trámites para viajar a Francia, país que inicialmente era su "destino" pero que, por no poder cumplir con todos los requerimientos para la visa en su momento, tuvo que desistir de viajar y optar por Argentina aunque, como él sostiene, "en realidad, Argentina nunca fue un lugar para venir ni para quedarme [...] Y ahora estaba pensando en ir a Francia. Sí, porque Francia siempre es un sueño. Sí, tengo que ir".

De este modo, se advierte cómo, para muchos de los jóvenes que actualmente residen en las ciudades de Córdoba y Rosario, los "destinos" de Estados Unidos, Canadá y Francia tienen una gran significación en sus idearios sobre la migración. De manera que, dada la relación histórica de la migración haitiana con esos países, las redes familiares existentes y las imágenes construidas en torno a ellos, se comprende que estos países continúen siendo aun los destinos más deseados por muchos. Como sostienen Salazar y Smart (2011), en un espacio global desigual, la construcción de los posibles lugares donde migrar, sea con base en conocimientos fundados o rumores se filtra a través de las aspiraciones personales de los migrantes. Es así que, si bien como parte de las posibilidades evaluadas para realizar los estudios universitarios también aparecen los países de República Dominicana, Cuba, México y, ya alejado en el tiempo, Venezuela, estos no llegan a ser descritos de la misma manera que los Estados Unidos, Francia y Canadá. Así, aunque la referencia a los "destinos ideales" al momento de definir los proyectos migratorios no es totalizante, es decir, no atraviesa la reconstrucción que se hace en todos los relatos, su significación —sea de manera crítica o no- aparece tanto en los que llegaron a mediados del 2000 como en aquellos que lo hacieron en los últimos años. Esto conduce a ponderar, no solo el peso gravitante que tienen estos "destinos ideales" sino también, y sobre todo, las diferentes miradas y estrategias que se configuran en torno a los mecanismos de control migratorio y fronterizo asociados a ellos y sus posibilidades de atravesamiento.

En esta dirección, es posible analizar las tensiones entre movilidad, fronteras y nacionalidad a partir de las dificultades percibidas para conseguir los visados requeridos para viajar a los Estados Unidos, Canadá o Francia. Dichas tensiones aparecen de manera particularmente nítida en la emergencia de las estrategias de movilidad hacia la Argentina que tienen lugar desde mediados de 2000 hasta, con distintas variantes, los años 2014, 2015 y 2016. Por esos años, como se verá en el siguiente apartado, las percepciones sobre el control fronterizo en Argentina y sobre lo que significa e implica la exención de visado para el ingreso al territorio comienzan a mutar significativamente. Así, si tomamos en primer lugar las experiencias y percepciones en torno a la movilidad de aquellos haitianos que llegaron antes de 2016, se advierte un claro contraste entre Argentina (y algunos países sudamericanos) y aquellos que se consideran los "destinos ideales" para migrar. Este contraste aparece de manera clara, por ejemplo, en una entrevista realizada a fines de 2017, en la que Lyne, una haitiana que arribó al país en 2005, señala lo que ella considera uno de los motivos por el cual muchos haitianos y haitianas eligen o eligieron Argentina como "destino": 
En Argentina, como en Chile o Brasil somos re nuevitos, porque recién hace dos años —o menos, o más- que Brasil por el tema del fútbol...antes sí o sí tenías que tener visa. Como necesitaban gente para trabajar, abrieron la puerta sin visa para que un haitiano pueda viajar. Todos los que tenían posibilidad de viajar aprovecharon para irse. Tanto ahora como Chile, que no hace falta tener visa, entonces aprovechan para viajar [...]. En cambio, en Estados Unidos o Canadá tenés que tener visa. Es muy difícil conseguirla. Entonces, por esa razón, si vos tenés un lugar que necesitás visa y otro que no, obviamente vas a optar por un lugar que vos no tenés que tener visa. Aparte, no solamente tenés que ir y hacer la demanda, tenés que pagar una suma que vos no sabés si te van a reconocer la visa o no, tanto para ir a Estados Unidos, como para Canadá o Francia. Aparte vos no sabés si te la van a dar o no. Es muy difícil que te la den. Entonces, en vez de gastar esos ochocientos dólares en ir a solicitar una visa sin saber si te la van a dar o no, ochocientos dólares ponés, por ahí un poco más, y te pagás un pasaje a Brasil, Chile o venir acá a la Argentina (Lyne, 15 de diciembre, 2017).

En otro caso, Emile, un haitiano que antes de venir a la Argentina en 2009 se había postulado para un programa de becas en Estados Unidos que finalmente no resultó, sostenía lo siguiente:

Y para Estados Unidos, si vos tenés a tus padres con residencia, podías viajar. Pero si tenías familiares que no tuvieran la ciudadanía no. [E: ¿Así es?] Sí. Primero tiene que solicitar una visa. Si tus padres tienen residencia, van a llenar la solicitud para ir a visitar y vivir con ellos. Muestran su ganancia...todo eso es un proceso largo. $Y$ hacen un filtro importante porque saben que si tus padres residían ahí vas a ir sí o sí. Pero te puede demorar años. [E: ¿Años?] Sí, porque es un proceso de desgaste también, lo hacen para que el haitiano siempre valore más ir...Para los haitianos Estados Unidos es un paraíso. En realidad, cuando lo pensás, no lo es.

A través de estos relatos, es posible comenzar a advertir algunas de las percepciones que tienen los jóvenes haitianos en relación al control migratorio y fronterizo en distintos países. En este sentido, las restricciones a la movilidad que se imponen a través de los visados se presentan desde el carácter selectivo de las fronteras, pero también como parte de las razones —entendidas en términos de "sentido práctico" (Bourdieu, 1997) - que conducen al desarrollo de nuevas estrategias de movilidad en las que se ponen en juego nuevos "destinos" y fronteras. Es en este escenario donde Argentina, un país que, hasta hace solo algunos años, era conocido únicamente por su fútbol o por la presencia de soldados argentinos a través de la MINUSTAH, emerge como un "destino posible", aunque no necesariamente "deseado". Como sostiene Robert:

(los haitianos) no sabían mucho de Latinoamérica porque de Argentina y Brasil sabemos que no son países desarrollados y también tienen sus problemas y quizás no nos pueden, entre comillas, mantener, y conocemos a Brasil y Argentina solo por su fútbol, pero últimamente vienen porque esa generación está desesperada, quiere un futuro mejor, quieren aventurarse y llegan acá, llegan acá y caen un montón de haitianos que piensan, como dije antes, que cualquier lugar en el mundo es mejor que Haití, no importa dónde están (Robert, 16 de noviembre, 2017). 
El desconocimiento sobre el país atraviesa de manera constante los proyectos de los jóvenes, así como también se multiplican los relatos sobre la forma, más o menos casual, en que Argentina emerge como "destino". Así, por ejemplo, en una entrevista grupal donde participaban dos haitianos y una haitiana, se produce el siguiente diálogo mientras uno de ellos, llegado a la Argentina en 2015, contaba cómo surge en su experiencia Argentina como lugar definido por su familia para viajar:

Y hablando, hablando... mi madre agarró su celular y llamó a uno de estos hermanos preguntándole cómo eran los trámites, qué tenían que hacer. Pero ya también, a pesar de que para estudiar en la Capital, Puerto Príncipe, también estaba por ahí la idea de ir a estudiar a Canadá. En Canadá están pidiendo más trámites. Pero cuando mi madre habló para Argentina, le decían que necesitaba una carta de invitación y prácticamente nada más. Y comprar su boleto.

Wilguens: ¡¿En serio?!

Michele: Claro, al lado de Estados Unidos...

Stéphane: Es como entrar al paraíso.

Wilguens: La solicitud de la visa creo que cuesta bastante...]

Michele: ¡Y no sabés si te la van a dar! [Ríen] (Wilguens, Michele y Stéphane, 7 de marzo, 2018).

La emergencia de Argentina como "destino" aparece de manera semejante en distintos relatos. De este modo, se advierte cómo la visa representa un elemento de restricción casi indiscutible a la hora de caracterizar el control de cualquier país y, por ello, se lo entiende como un elemento fundamental para las estrategias de movilidad o, al menos, de aquellos proyectos que son pensados en términos de "legalidad". Es decir, la importancia del visado es entendida en relación con las particularidades que se mencionaban antes: se trata de proyectos familiares orientados a la realización de estudios universitarios y, por lo tanto, los ingresos a los países extranjeros son pensados desde opciones administrativas contempladas por las distintas normativas migratorias. De este modo, la construcción diferenciada —y jerárquica- de "destinos" presente en las miradas de estos jóvenes contrasta con las posibilidades reales de ingresar a los países más anhelados, lo que produce una distancia entre el "destino deseado" y el "destino posible". En el examen de estas posibilidades las fronteras juegan un rol fundamental que es definido, en buena medida, por la existencia o no de visados para el ingreso. En este sentido, en muchos casos, Argentina como "destino" de los proyectos migratorios familiares de los jóvenes haitianos se construye por oposición a países como Estados Unidos, Canadá y Francia a partir de la imposibilidad percibida para conseguir los visados necesarios para su admisión o de experiencias personales de rechazos de visa. No obstante, como se verá a continuación, la exención de visado vigente en Argentina hasta agosto de 2018 no supuso la admisión irrestricta al territorio para nacionales haitianos y, en este sentido, se considera relevante analizar el desarrollo de sus estrategias de movilidad con base en las diferentes temporalidades de sus trayectorias. 


\section{Arribos recientes, ¿cambios en los esquemas de control migratorio y fronterizo?}

Ellos (los agentes migratorios) tienen una definición especial de lo que es ser turista [...], tiene que ser europeo o estadounidense, para ellos sí, ellos son turistas. Pero mientras tengas el pasaporte de la República haitiana ya no eres turista, o algunos países de África, ya no eres turista, ya vienes a hacer otra cosa, no eres turista (Fátima, 10 de abril, 2018).

La visa entendida como deslocalización del control fronterizo que permite al Estado participar en la preselección de los viajeros para disuadir la llegada de nacionales considerados no deseables (Bigo y Guild, 2017; Neumayer, 2006; Salter, 2006) pareciera cobrar materialidad en el modo en que para muchos jóvenes emerge Argentina como "destino"; es decir, a partir de la ponderación de la existencia o no de visado con respecto a otros países considerados como "destinos ideales". En este sentido, puede advertirse cómo la movilidad de haitianos aparece estructurada, al menos en parte, a partir de lo que algunos autores han denominado "régimen global de visados" (Salter, 2006; Salter y Mutlu, 2010). Como muestran diversas publicaciones sobre los datos de Passport Index ("Estos son los pasaportes", 2019; McKirdy, 2019; Yu, 2016), Haití es una de las nacionalidades con mayores restricciones de ingreso a países extranjeros en el mundo. En 2017 el pasaporte haitiano era admitido en 53 países, pero solamente en 17 de ellos sin necesidad de visa o algún otro tipo de documento. Entre estos 17 países se incluían Chile y Argentina que implementaron visados consulares para haitianos durante el año 2018. En este punto, además de entender las restricciones en los visados como una forma diferencial y selectiva de control que contribuye a reforzar las desigualdades sociales entre países y entre sujetos (Neumayer, 2006), se considera necesario reparar en el proceso histórico que configura la política de visado para nacionales haitianos en Argentina a partir de las diferentes formas en que los sujetos experimentan, disputan o negocian las prácticas de control. Como se explicitaba al inicio, esto supone considerar no solo las lógicas gubernamentales de control, sino también las prácticas de los sujetos migrantes (Casa-Cortes, Cobarrubias, De Genova et al, 2015).

En particular, interesa destacar las tensiones que se producen entre los rechazos en frontera bajo la categoría de "falso turista" y las estrategias de movilidad de haitianos hacia la Argentina a lo largo de distintas trayectorias. Como se había mencionado, la conformación de Argentina como "destino" y las estrategias de movilidad hacia el país muestran diferencias relevantes según la temporalidad de las distintas trayectorias. Es así que, aunque la exención de visado para haitianos en la Argentina se mantiene vigente hasta agosto de 2018, no será lo mismo la experiencia de cruzar la frontera para alguien que llegó a mediados del 2000 que para alguien que llegó de fines de 2016 en adelante. Tampoco lo será para aquellos que llegaron en los años posteriores al terremoto de 2010, momento en que ya habían comenzado a circular ciertos idearios y prácticas humanitarias específicas sobre este grupo nacional. De este modo, se observan importantes matices no solo en lo que respecta al conocimiento que se tenía del país según el año de llegada (en parte, facilitado por redes más consolidadas y el acceso a las nuevas tecnologías), sino también en relación con los cambios en las regulaciones estatales referidas al ingreso y admisión al territorio nacional argentino y, las diferentes 
experiencias de cruce de frontera en los aeropuertos de Argentina. Con todo, esto no implica sugerir que las prácticas de control y vigilancia hubieran estado ausentes antes del 2016, ni que el visado de turismo para haitianos — y los rechazos en frontera que le fueron co-constitutivos - sean producto de una transformación en los esquemas de control migratorio y fronterizo que tendrían lugar en concomitancia con el cambio de gobierno nacional en 2015. ${ }^{4}$ En Sudamérica, los avances en las tecnologías de seguridad y vigilancia aplicadas al control migratorio han sido señaladas en distintos trabajos (Domenech, 2013; 2017; Santi, 2018). En Argentina, en particular, se considera que la introducción del uso de la biometría en 2011, los sistemas de Información Anticipada de Pasajeros (API) y Registro de Nombres de Pasajeros (PNR) en los aeropuertos desde 2014 (Resolución 618/2014, 2014), y la creación de la Comisión Nacional de Fronteras en 2017 (Decreto 68/2017, 2017), son fundamentales para comprender el desarrollo de los controles migratorios y fronterizos. Asimismo, cabe señalar que el uso de la figura de falso turista en Argentina tiene un recorrido histórico trazado que va desde la primera normativa en 1985 hasta su última modificación en 2014 a través de la Resolución 4362/2014 (Disposición No 4362/2014, 2014; Alvites, 2018). En este sentido, no resulta un dato menor que en 2014, por ejemplo, se presentaran para ingresar al país 2374 haitianos de los cuales fueron rechazados 208. Es decir, 8,7\% no fue admitido en el territorio argentino. Aunque el análisis no pretende fundamentarse en datos cuantitativos, resulta significativo mencionar que luego de una disminución de los rechazos en frontera de personas de nacionalidad haitiana en 2016 con 59 rechazos, en 2017 se registraron 221 casos y, hasta agosto de 2018, cuando se impone el visado de turismo, ya se contabilizaban 900 casos (Trabalón, 2018).

Así, sin desconocer el sesgo restrictivo y criminalizante que acompaña a las diferentes regulaciones normativas, prácticas y discursos oficiales en materia de política migratoria del gobierno de Cambiemos, se sostiene que las lógicas de control que subyacen a la política de visado, los rechazos y las experiencias de los sujetos en torno y a través de ellas, pueden ser mejor comprendidas si se inscriben en el proceso de regionalización de la política migratoria (Domenech, 2013; 2017) y, en un sentido más general, en el marco de la conformación de un régimen global de migraciones y fronteras (Mezzadra, 2012). Estos desarrollos brindan un campo analítico más propicio para entender la imbricación de las diferentes escalas locales, nacionales, regionales y globales (Glick-Schiller y Caglar, 2010; Feldman-Bianco, 2015, 2018) que operan en las diversas prácticas y experiencias de control a través de los efectos que producen las fronteras y categorías nacionales (Sayad, 2008), pero también más allá de estas.

En este escenario, se conectan las experiencias de los y las jóvenes haitianos y haitianas hacia Argentina que tienen lugar desde mediados del 2000 con la reconfiguración de las prácticas de control migratorio y fronterizo, y el aumento en las restricciones de visados para determinados grupos nacionales en el espacio

\footnotetext{
${ }^{4}$ Siguiendo el planteo de Domenech (2017), desde mediados del 2000, diferentes transformaciones y sucesos particulares contribuyeron a instalar una representación dominante en el campo de las políticas migratorias asociada a una supuesta excepcionalidad sudamericana con respecto a los esquemas de control y vigilancia que se observaban en otras partes del mundo. En contraposición a esta mirada, que sostiene que se habría producido el paso desde un enfoque de seguridad y control a uno de derechos humanos, se propone reconocer "la coexistencia entre procesos y acontecimientos regionales específicos y las prácticas de control y vigilancia que operan en el presente en distintos países, diversos espacios, a diferentes escalas y a través de numerosos actores en el ámbito regional sudamericano" (Domenech, 2017, p. 38).
} 
sudamericano. Ciertamente, durante este periodo es posible advertir procesos de reforzamiento fronterizo que tienen lugar en y entre diferentes países sudamericanos (Alvites, 2019; Domenech, 2013, 2017; Ramírez y Alfaro, 2010), así como el desarrollo de tecnologías de seguridad y vigilancia aplicadas al control migratorio (Domenech, 2017; Santi, 2018). Además, la implementación de visados consulares se encuentra estrechamente ligada a la aparición de nuevos movimientos y patrones migratorios, tal como lo demuestran los que se han implementado a grupos nacionales del Caribe, Asia y África como también, de manera más reciente, a los nacionales venezolanos. Entre algunos ejemplos recuperamos dos de distintos informes (orm, 2016; 2017; CEPAL, 2018) aparece el caso de Ecuador que, luego de la medida de suspensión de visas en 2008, el gobierno reimpone los visados consulares a nacionales de Eritrea, Etiopía, Kenia, Nigeria y Somalia en el año 2010 y, a Senegal y Cuba, en el año 2015. De manera similar, tras haber sido suspendidos los visados consulares para ciudadanos chinos, en Ecuador en 2008 y en Colombia en 2007, al poco tiempo son nuevamente reimpuestos en ambos países. En relación con los nacionales de República Dominicana se establecieron visados de turismo en el año 2012 en Argentina y Chile, y en el año 2014 en Uruguay. En este último país también se implementó un visado de turismo para cubanos en 2015. Con respecto a los nacionales haitianos, se reglamentaron visados de turismo en 2012 en Perú, en abril de 2018 en Chile, y en agosto de 2018 en Argentina. Además, para este grupo nacional se disponen para los haitianos visados humanitarios en 2012 en Brasil y en 2018 en Chile por motivos de reunificación familiar, y preavisos de viaje en 2015 en Ecuador. Actualmente, una tendencia similar se observa en la regulación de los movimientos de venezolanos en la región, para quienes se pasó a exigir visados humanitarios en Perú y Ecuador, y visado de turismo en Chile (Cepal, 2018; оIM, 2016).

Si bien con matices específicos, la política migratoria argentina no es ajena a estos procesos regionales y globales. Es posible constatarlo al considerar, entre otros elementos, cómo se han configurado los rechazos y visados en los últimos años a partir de determinados perfiles de viajeros y la acentuación del trato diferencial entre las categorías de migrantes Mercosur y Extra Mercosur, su constitución y fundamentación a partir de argumentos securitarios y humanitarios y el desarrollo particular que ha tenido la aplicación de la figura de falso turista para los movimientos de nacionales haitianos desde mediados del 2000 al presente.

En este escenario, el incremento de la violencia estatal que se observa en la actual coyuntura política del gobierno nacional se conjuga con procesos más generales asociados a la estructuración de las desigualdades y movilidades a escala global (Feldman-Bianco, 2018). Así, se entiende que, por ejemplo, muchos de los y las jóvenes haitianos y haitianas perciban cambios en la orientación de la política migratoria y, al mismo tiempo, que consideren que ser rechazados en Argentina haya sido siempre una posibilidad latente y concreta. Como señala Joseph, que llegó al país en 2011:

También está difícil acá. Cualquier haitiano que está acá en Argentina, antes de viajar o al momento de viajar, o sea, los chicos dicen siempre, "es un viaje 50,50 ”, 50 podés entrar y 50 te pueden mandar de vuelta. Me pasó lo mismo también cuando viajé. Viajaba y dije, "Bueno, en cualquier momento me pueden echar" (Joseph, 17 de noviembre, 2017).

Asimismo, también es cierto que, para muchos, la significación que adquiere el visado y los rechazos no puede ser comprendida fuera de la coyuntura política argentina y 
de determinadas prácticas, normativas y discursos promovidos por el gobierno actual, ${ }^{5}$ asociados también, en varios casos, a la crisis económica que atraviesa el país. En este marco, desde inicios del trabajo de campo, en diciembre de 2017, una de las primeras cuestiones que emergía por parte de los jóvenes en las conversaciones era el tema del ingreso y, más precisamente, los problemas que estaban teniendo aquellos haitianos y haitianas que llegaban a la Argentina para ser admitidos en el país. A lo largo del 2018 esta situación fue adquiriendo mayor notoriedad a medida que se observaba, de manera cada vez más preocupante, un aumento progresivo de los rechazos en frontera de aquellos conocidos, amigos y familiares que intentaban ingresar al país a través de los diferentes aeropuertos de Argentina. De esta manera, hasta la implementación del visado de turismo para nacionales haitianos en agosto de 2018, las prácticas de cruce de frontera y los rechazos por falso turista ${ }^{6}$ se convirtieron en elementos centrales que atravesaron la cotidianeidad de los migrantes haitianos y haitianas residentes en las ciudades de Rosario y Córdoba. De las relaciones corrientes mantenidas con algunos jóvenes se pudo advertir cómo al inicio del trabajo de campo, cuando se tematizaba sobre los rechazos en frontera, había opiniones divididas entre quienes miraban de manera crítica estos rechazos, los que lo entendían como un ejercicio legítimo de la soberanía de Estado (muchas veces atribuida a la falta de los requisitos adecuados por parte de los propios viajeros) y quienes, si bien los identificaban como un problema, mostraban cierta tolerancia, como si se tratase de un riesgo admisible o válido para aquellos viajeros que emprenden sus experiencias migrantes hacia otros países. Sin embargo, con el pasar del tiempo, fue posible ir observando cómo, para muchos, este piso de tolerancia comenzaba a resquebrajarse a medida que se percibía con mayor evidencia que los rechazos a haitianos y haitianas en los aeropuertos eran de carácter sistemático y discrecional.

En este marco, a través de las diferentes miradas y experiencias de cruce de frontera, detención y rechazo, interesa recuperar cómo interviene el componente racial y étnico ligado al origen nacional en la configuración de las prácticas de control en diferentes aeropuertos de Argentina y en las reconfiguraciones de frontera para nacionales haitianos hacia este país. En tal sentido, en diferentes momentos se relataron situaciones en las que, por ejemplo, al bajar del avión y hacer la fila de migrantes Extra Mercosur en el aeropuerto de Ezeiza, los agentes migratorios se acercaban para separar a aquellos que fueran "negros" en una fila aparte, según Emile: "nos separan primero por ser negros, después por nuestra nacionalidad", mientras que para Fátima "por nuestro color de piel nos consideran diferentes como si fuéramos otra cosa", y para Carl "como somos negros y un negro no es algo que se quiere para ningún país". Al mismo tiempo, otro de los hechos recurrentes en los cruces de frontera remitía a cómo eran interpelados por los agentes migratorios a partir de la asociación entre la pobreza de Haití y la imposibilidad, debido a eso, de que las personas de nacionalidad haitiana pudiesen ser consideradas como turistas, como sostenía Moise:

\footnotetext{
${ }^{5}$ Un panorama detallado de los cambios normativos y determinados discursos y prácticas en la actual coyuntura del gobierno de Cambiemos puede verse, por ejemplo, en Canelo, Gavazzo y Nejamkis (2018) y García y Nejamkis (2018).

${ }^{6}$ La figura de falso turista no aparece inmediatamente junto a los rechazos, en parte, porque los propios haitianos y haitianas que eran rechazados no tenían conocimiento del porqué del impedimento de ingreso a la Argentina y posterior reconducción a Haití.
} 
¿Y sabés lo que te dicen, la pregunta? "Conocemos la situación de Haití, no me digan que vienen como turista acá a la Argentina, decinos la verdad, que vienes para estar, para vivir, para hacer otra cosa”. Es decir que ponen directamente que un haitiano no puede venir como turista a la Argentina. [...] Por ser haitiano, por la situación del país. Miran la situación del país y te lo ponen al frente. La situación de tu país no te permite entrar como turista [...] (Moise, 6 de abril, 2018).

Estos relatos ponen en evidencia algunas de las formas en que interviene el componente racial y étnico en las prácticas estatales de control migratorio y fronterizo. Como se planteó al inicio, las prácticas de racialización en Argentina derivan tanto del sistema colonial europeo como de la formación histórica de la "argentinidad". En esta línea, el racismo como "fenómeno social total" (Wallerstein y Balibar, 1991) existe y persiste como dispositivo que apunta a postular las razas y, en

[...] última instancia se trata de la persistencia de la racialización que, en tanto proceso cognitivo y valorativo sostenido por y sostenedor de relaciones de poder, construye en "dato" biológico de la existencia de las razas que da sustento al racismo (Caggiano, 2008, p. 34).

De tal forma, el cruce entre migración, racialización y etnicidad debe realizarse en el marco de la construcción de los idearios de una nación "sin negros" y "sin indios" y, también, a partir de la forma en que se reconfiguran las "fronteras raciales" (Fassin, 2011) en Argentina desde mediados del 2000 a partir del aumento de la visibilidad de migrantes africanos y afrodescendientes, como parte de los nuevos movimientos y patrones migratorios que se observan en las últimas dos décadas en la región.

En este escenario, se considera que el análisis realizado arroja luz sobre dimensiones claves para la comprensión de las prácticas y experiencias de control en Argentina. La violencia material y simbólica ejercida a través de los controles fronterizos y rechazos de haitianos en diferentes aeropuertos tiene lugar a partir de la mediación fundamental que opera a través de los marcadores raciales y étnicos ligados a la nacionalidad -y una supuesta clase social一. Así, en los controles fronterizos la identificación racial precede a la étnica, pero son ambas las que, ligadas al origen nacional, terminan por configurar aquella condición social que remite o se materializa a través de la figura del migrante como construcción diferenciada del extranjero y, por lo tanto, de turista. Siguiendo el análisis de Sayad:

[...] inmigrante designa cada vez con mayor frecuencia una condición social mientras que extranjero corresponde a un estatus jurídico-político; este último puede cambiar sin que nada cambie en la primera o sin que cambie nada en absoluto. Si todos los extranjeros no son (socialmente hablando) inmigrantes, todos los inmigrantes no son necesariamente extranjeros (jurídicamente hablando) (Sayad, 2008, p. 103).

\footnotetext{
${ }^{7}$ Mientras que los procesos de etinicización han sido abordados en relación con la migración limítrofe por algunos autores desde los noventa a la actualidad (Caggiano, 2005; Grimson, 2010), los procesos de racialización son más incipientes, en parte, porque la migración de africanos y afrodescendientes comenzó a adquirir mayor visibilidad en Argentina desde mediados del 2000, siendo la migración africana, y senegalesa en particular, la más prolífica.
} 
De este modo, los marcadores raciales, étnicos y nacionales posibilitarían la conversión de viajeros o turistas a potenciales migrantes y, en consecuencia, a posibles riesgos o amenazas al orden nacional. En esta línea, la categoría de turista, y su contraparte falso turista pueden ser interpretadas a partir de este régimen diferencial de extranjería a través de la cual algunos extranjeros permanecen como tales mientras otros pasan a ser considerados como migrantes o, para el caso, potenciales migrantes. Dicho régimen atraviesa las prácticas fronterizas que conciernen los ingresos y admisiones, sea a través de formas de "control remoto" (Zolberg, 2003) como los visados o en los cruces de fronteras de los aeropuertos. En este sentido, se destacan las formas particulares de control que configuran al inmigrante como "portador del estatuto y posición atribuida a su país en la escala internacional (Sayad, 1998) y, al mismo tiempo, develan la producción estatal de fronteras raciales (Fassin, 2011) en las formas de regulación, vigilancia y detención de las movilidades en los aeropuertos de Argentina.

\section{Conclusiones}

Diferentes estudios sobre políticas de visados muestran cómo las restricciones de visados producen un sistema altamente desigual a través del cual se alienta la movilidad transnacional de aquellos titulares de pasaportes de países privilegiados (ricos) a expensas de severas restricciones que buscan disuadir la llegada de aquellos considerados indeseables. Así, a través de un régimen de acceso diferencial a los espacios extranjeros se refuerzan las desigualdades ya existentes. En este sentido, las políticas de visados se presentan como una forma de regulación fundamental para la estructuración de la movilidad que ofrece la posibilidad de comprender aquellos mecanismos de control que operan fuera del territorio nacional, pero también cómo estos se constituyen en el marco de diversos procesos de configuración de las fronteras, en los que intervienen diferentes actores, prácticas, discursos y, en particular, los propios movimientos migratorios. Así, esta primera aproximación a las políticas de visados para haitianos en Argentina y la región permitió no solo mostrar cómo en la última década las fronteras se han modificado sustancialmente para este grupo nacional, sino también reparar en algunos aspectos fundamentales de sus experiencias migrantes. Para el caso de las trayectorias de haitianos hacia las ciudades de Córdoba y Rosario es posible advertir los vínculos que se establecen entre la imposibilidad de obtener los visados a ciertos destinos y la puesta en marcha de estrategias de movilidad que incluyen la búsqueda de nuevos horizontes. También se observa el uso estratégico de ciertas categorías como la de turistas a lo largo de las distintas trayectorias, las experiencias de cruce y las reconfiguraciones que se dan en el tiempo en materia de control estatal.

De esta manera, el visado para nacionales haitianos es considerado como una práctica fronteriza que, definiéndose en el marco de las políticas de movilidad dominantes, opera como un mecanismo de regulación correctivo una vez que las estrategias de movilidad de haitianos hacia la Argentina, a través de las porosidades en las fronteras, devienen en factor problemático. Así, lo que normativamente se plasma en el visado de turismo en agosto de 2018 es resultado de un proceso histórico en que confluyen diversos actores, discursos y prácticas. La figura del falso turista en 
particular opera como categoría estatal a partir de una construcción social previa de la figura del migrante. El migrante designa una condición social inextricablemente asociada a una idea de sospecha que permite al Estado, basado en criterios raciales y étnicos de control, equipararlo con un sujeto peligroso, o al menos riesgoso, para el orden nacional y que, por lo tanto, debe ser - y es- legítimamente rechazado. En este escenario, entender la frontera como relación social permite abordar su carácter histórico, heterogéneo y móvil. Así, las fronteras aparecen en la configuración misma de los proyectos migratorios de estos jóvenes y en la puesta en marcha de sus estrategias de movilidad hacia la Argentina. Pero también en la cotidianeidad misma, en la ruptura de lazos familiares y sociales, y en las sensaciones de objeción o impugnación de su presencia provocada, por ejemplo, por los rechazos en frontera y la normativa del visado de turismo.

\section{Referencias}

Alarcón, M. (2015). Las asociaciones de migrantes haitianos en el Ecuador: Entre debilidad y resistencia. REMHU. Revista Interdisciplinar da Mobilidade Humana, 23(44), 207-220. Recuperado de http:/ /www.redalyc.org/articulo.oa?id=407042021013\}

Alvites, A. (2018). Extranjeros bajo la lupa: La figura del "falso turista" en Argentina. Horizontes Decoloniales, 4, 39-62.

Alvites, A. (2019). Migraciones internacionales, fronteras y Estados. ¿Cómo interpretar el régimen de frontera desde América del Sur? Desafios, 31(1), 123-156.

Balibar, E. (2012). Politics and the other scene. Londres-Nueva York: Verso Trade.

Berganza, I. (2017). Los flujos migratorios mixtos en tránsito por Perú: Un desafío para el Estado. En N. Pedemonte y J. Koechlin (Eds.), Migración haitiana hacia el sur andino (pp. 41-64). Santiago, Chile: овıмid.

Bigo, D. y Guild, E. (2017). Policing at a distance: Schengen visa policies. En D. Bigo. (Ed.), Controlling Frontiers (pp. 233-263). Londres, Inglarerra: Routledge.

Bourdieu, P. (1997). Razones prácticas. Sobre la teoría de la acción. Barcelona, España: Anagrama.

Bourdieu, P. (2011). Las estrategias de reproducción social. Buenos Aires, Argentina: Siglo XXI.

Burey, R. (2018). Estrategias migratorias en el tránsito de emigrantes haitianos hacia Estados Unidos. Huellas de la Migración, 2(4), 93-123. Recuperado de https:// huellasdelamigracion.uaemex.mx/article/view/4604/8005

Caggiano, S. (2005). Lo que no entra en el crisol: Inmigración boliviana, comunicación intercultural y procesos identitarios. Buenos Aires, Argentina: Prometeo Libros.

Caggiano, S. (2008). Racismo, fundamentalismo cultural y restricción de la ciudadanía: formas de regulación social frente a inmigrantes en Argentina. En S. Novick (Comp.). Las migraciones en América Latina. Políticas, culturas y estrategias (pp. 31-51). Buenos Aires, Argentina: Catálogos-S.R.L.-CLACso-Asdi.

Canelo, B., Gavazzo, N. y Nejamkis, L. (2018). Nuevas (viejas) políticas migratorias en la Argentina del cambio. Si Somos Americanos, 18(1), 150-182. Recuperado de https://scielo.conicyt.cl/scielo.php?pid=S0719-09482018000100150\&script=sci_arttext 
Casas-Cortes, M., Cobarrubias, S., De Genova, N. et al. (2015). New Keywords: Migration and Borders. Cultural Studies, 29(1), 55-87. doi:10.1080/09502386.2014.891630

Casas-Cortes, M., Cobarrubias, S. y Pickles, J. (2015). Riding routes and itinerant borders: Autonomy of migration and border externalization. Antipode, 47(4), 894914.

Ceja, I. (2015). Migraciones haitianas en la región andina (Dossier central). Andina Migrante, (19), 2-13. Recuperado de http://repositorio.flacsoandes.edu.ec/ bitstream/10469/6772/1/BFLACSO-AM19-01-Ceja.pdf

Cepal. (2018). Panorama de la migración internacional en América del Sur. Cepal-Serie población y desarrollo, (123). Chile: Cepal.

De Heusch, F. (2016). "Amo a mi país, sigo hablando en creole, pero tomo el fernet con coca y como el asado": Una etnografía de los migrantes haitianos y de la venta de "bijouteria" en la ciudad de Córdoba, Argentina (Tesis de licenciatura). Universidad Nacional de Córdoba, Córdoba, Argentina.

Domenech, E. (2007), La agenda política sobre migraciones en América del Sur: el caso de la Argentina. Revue Européenne des Migrations Internationales, 23(1), 71-94

Domenech, E. (2013). "Las migraciones son como el agua": Hacia la instauración de políticas de "control con rostro humano". La gobernabilidad migratoria en la Argentina. Polis Revista Latinoamericana, 12(35), 1-21.

Domenech, E. (2017). Las políticas de migración en Sudamérica: Elementos para el análisis crítico del control migratorio y fronterizo. Terceiro Milênio. Revista Crítica de Sociologia e Política, 8(1), 19-48. Recuperado de https://ri.conicet.gov.ar/ handle/11336/58156

Duffard, I. (2016). Del Caribe haitiano a la Argentina: Trayectorias de cuerpos en movilidad humana pos terremoto 2010. Recuperado de http://biblioteca.clacso.edu.ar/clacso/pobreza/20160307040904/Duffard.pdf

Düvell, F. (2003). The Globalisation of Migration Control. London. Recuperado de https:// www.opendemocracy.net/en/globalisation-of-migration-control/

Estos son los pasaportes más poderosos de América Latina. (3 de julio de 2019). CNN en español. Recuperado de https://cnnespanol.cnn.com/2019/07/03/estos-son-los-pasaportes-mas-poderosos-de-america-latina/

Fassin, D. (2011). Policing Borders, Producing Boundaries. The Governmentality of Immigration in Dark Times. Annual Review of anthropology, 40, 213-226. Recuperado de https://www.annualreviews.org/doi/10.1146/annurev-anthro-081309-145847

Feldman-Bianco, B. (2015). Desarrollos de la perspectiva transnacional: migración, ciudad y economía política. Alteridades, 25(50), 13-26.

Feldman-Bianco, B. (2018). Anthropology and ethnography: the transnational perspective on migration and beyond. Etnográfica. Revista do Centro em Rede de Investigação em Antropologia, 22(1), 195-215. Recuperado de https://www.academia. edu/36658151/Etnogr\%C3\%A1fica_Revista_do_Centro_em_Rede_de_Investiga\%C3\%A7\%C3\%A3o_em_Antropologia

Fernandes, D. y Faria, A. (2017). O visto humanitário como resposta ao pedido de refúgio dos haitianos. Revista Brasileira de Estudos de População, 34(1), 145-161. Recuperado de http://www.scielo.br/scielo.php?pid=S010230982017000100145\&script $=$ sci_abstract\&tlng=es 
García, L. y Nejamkis, L. (2018). Regulación migratoria en la Argentina actual: del "modelo" regional al recorte de derechos. Autoctonía. Revista de Ciencias Sociales e Historia, 2(2), 219-241.

Glick-Schiller, N. y Caglar, A. (2010). Introduction: Migrants and Cities. En N. Glick-Schiller y A. Caglar (Eds.), Locating migration: Rescaling cities and migrants (pp. 1-22). Nueva York, Estados Unidos: Cornell University Press.

Glick-Schiller, N. y Salazar, N. (2013). Regimes of mobility across the globe. Journal of Ethnic and Migration Studies, 39(2), 183-200.

Grimson, A. (2010). Relatos de la diferencia y la igualdad: los bolivianos en Buenos Aires. Buenos Aires, Argentina: Eudeba.

Guild, E. y Bigo, D. (2003). Le visa Schengen: expression d'une stratégie de «police» à distance. Cultures \& Conflits, (49), 22-37.

Levitt, P. y Glick-Schiller, N. (2004). Perspectivas internacionales sobre migración: conceptuar la simultaneidad. Migración y Desarrollo, (3), 60-91.

McKirdy, E. (3 de julio de 2019). Los pasaportes más poderosos del mundo a mediados de 2019. CNN en español. Recuperado de https://cnnespanol.cnn.com/2019/07/03/ los-pasaportes-mas-poderosos-del-mundo-a-mediados-de-2019/

Mezzadra, S. (2012). Capitalismo, migraciones y luchas sociales: la mirada de la autonomía. Nueva Sociedad, (237), 159-178. Recuperado de https://nuso.org/media/articles/downloads/3826_1.pdf

Mezzadra, S. y Neilson, B. (2017). La frontera como método. Madrid, España: Traficantes de sueños.

Neumayer, E. (2006). Unequal Access to Foreign Spaces: How States Use visa Restrictions to Regulate Mobility in a Globalized World. Transactions of the Institute of British Geographers, 31(1), 72-84. Recuperado de https://rgs-ibg.onlinelibrary. wiley.com/doi/10.1111/j.1475-5661.2006.00194.x

Nieto, C. (2014). Migración haitiana a Brasil: Redes migratorias y espacio social transnacional. Recuperado de http://biblioteca.clacso.edu.ar/ clacso/se/20141118015558/ Migracion.pdf

овIмID. (2016). La migración en Chile: breve reporte y caracterización. Informe del Observatorio Iberoamericano sobre Movilidad Humana, Migraciones y Desarrollo. Recuperado de https://drive.google.com/file/d/0Bw1mkAYjY1DzWWhibFpQUmNyWWs/view

OIM. (2017). Recientes tendencias migratorias extra e intra-regionales y extra-continentales en América del Sur. Informe migratorio Sudamericano. Recuperado de https://robuenosaires.iom.int/sites/default/files/Documentos\%20PDFs/Recientes_tendencias_migratorias_extra_e_intra_regionales_y_extra_continentales_en_america_del_sur_es.pdf

OIM-FCCAM. (2017). Estudio exploratorio sobre las trayectorias socio-educativas y socio-laborales de migrantes haitianos, senegaleses y ucranianos en la ciudad de Buenos Aires. Buenos Aires. oIm. Recuperado de http://argentina.iom.int/co/estudio-exploratorio-sobre-las-trayectorias-socio-educativas-y-socio-laborales-de-migrantes

OIM-OEA. (2016). Informe Regional "Flujos de migrantes en situación migratoria irregular provenientes de África, Asia y el Caribe en las Américas". Recuperado de http://www.oas.org/es/sadye/publicaciones/Reporte-OIM_OEA_SPA.pdf 
Papadopoulos, D. y Tsianos, V. (2013). After Citizenship: autonomy of migration, organisational ontology and mobile commons. Citizenship Studies, 17(2), 178-196. doi:https://doi.org/10.1080/13621025.2013.780736

Pedemonte, N., Amode, N. y Rencoret, J. (2015). Racismo y matrices de "inclusión" de la migración haitiana en Chile: elementos conceptuales y contextuales para la discusión. Polis. Revista Latinoamericana, 14(42), 217-245.

Pedemonte, N. Amode, N. y Vásquez, J. (2017). Migración haitiana hacia Chile: origen y aterrizaje de nuevos proyectos migratorios. En N. Pedemonte y J. Koechlin (Eds.), Migración haitiana hacia el sur andino (pp. 65-172).

Quijano, A. (2000). Colonialidad del poder, eurocentrismo y América Latina. En E. Lander (comp.), La colonialidad del saber: Eurocentrismo y ciencias sociales. Buenos Aires: CLACSO.

Ramírez, J. y Alfaro, Y. (2010). Espacios multilaterales de diálogo migratorio: El proceso Puebla y la Conferencia Sudamericana de Migración. Andina Migrante, 9, 2-10. Recuperado de https://repositorio.flacsoandes.edu.ec/handle/10469/2769

Salazar, N. y Smart, A. (2011). Anthropological takes on (Im) mobility. Identities, 18(6), i-ix.

Salmón, M. (2017). Tránsitos migratorios irregularizados en América Latina. Flacso Ecuador. (Dossier Central). Andina Migrante, 2, 2-9.

Salter, M. (2006). The Global Visa Regime and the Political Technologies of the International Self: Borders, bodies, biopolitics. Alternatives, 31(2), 167-189. doi: $10.1177 / 030437540603100203$

Salter, M. y Mutlu, C. E. (2010). Asymmetric borders: The Canada- Czech Republic 'visa war' and the question of rights. CEPS. Liberty and Security in Europe. Recuperado de http://aei.pitt.edu/15127/1/Salter_\&_Mutlu_on_Asymmetric_Borders. pdf

Santi, S. (2018). Biometría y vigilancia social en Sudamérica: Argentina como laboratorio regional de control migratorio. Revista Mexicana de Ciencias Políticas y Sociales, 232(63), 247-268. Recuperado de http://www.scielo.org.mx/scielo. php?pid=S0185-19182018000100247\&script=sci_arttext

Sassen, S. (2007). Una sociología de la globalización. Buenos Aires, Argentina: Katz editores.

Sayad, A. (1998). A imigracão ou os paradoxos da alteridade. San Pablo: EDUSP.

Sayad, A. (2008). Estado, nación e inmigración. El orden nacional ante el desafío de la inmigración. Apuntes de Investigación del CECYP, (13), 101-116.

Segato, R. L. (2007). La nación y sus otros: Raza, etnicidad y diversidad religiosa en tiempos de políticas de la identidad. Buenos Aires, Argentina: Prometeo Libros.

Segato, R. (2011). Racismo, discriminación y acciones afirmativas: Herramientas conceptuales. Observatório da Jurisdição Constitucional, 1(1), 1-16.

Silva, S. (2015). Fronteira amazónica: passagem obrigatória para haitianos? REMHU. Revista Interdisciplinar da Mobilidade Humana, 23(44), 119-134. Recuperado de http:/ / www.scielo.br/Scielo.php?pid=S198085852015000100119\&script=sci_ abstract\&tlng=pt

Thomaz, D. Z. (2013). Migração haitiana para o Brasil pós-terremoto: Indefinição normativa e implicações políticas. Primeiros Estudos, (4), 131-143. Recuperado de: http://www.revistas.usp.br/primeirosestudos/article/view/56732 
Torpey J. (1998) Coming and Going: on the State Monopolization of the legitimate "Means of Movement". Sociological Theory, 16(3) 239-259.

Trabalón, C. I. (2018). Política de visado y regulación de las fronteras. Un análisis desde la movilidad de haitianos en Sudamérica. Polis. Revista Latinoamerica (Santiago), 17(51), 163-186.

Véran, J., Da Silva, D. y Fainstat, T. (2014). Nem refugiados, nem migrantes: a chegada dos haitianos à cidade de Tabatinga (Amazonas). Dados. Revista de Ciências Sociais, 57(4), 1007-1041. Recuperado de http://www.redalyc.org/ $\mathrm{html} / 218 / 21835768005 /$

Vila, P. (2000). Crossing borders, reinforcing borders: Social categories, metaphors, and narrative identities on the US-Mexico frontier. Austin: University of Texas Press.

Wallerstein, I. y Balibar, E. (1991). Raza, nación y clase. Madrid, España: IEPALA Editorial.

Yu, E. (1 de marzo de 2016). Los mejores y los peores pasaportes del mundo. CNN español. Recuperado de https://cnnespanol.cnn.com/2016/03/01/los-mejores-y-los-peores-pasaportes-del-mundo/

Zolberg, A. (2003). The archaeology of "remote control". En A. Fahrmeir, O. Faron y P. Weil (Eds.), Migration control in the North Atlantic world: The evolution of state practices in Europe and the United States from the French revolution to the inter-war period (pp. 195-222). Nueva York: Berghahn Books

\section{Material legislativo}

Decreto 68/2017. (2017). Comisión Nacional de Fronteras, 25 de enero de 2017. Buenos Aires, Argentina. Recuperado de https://www.boletinoficial.gob.ar/detalleAviso/primera/158309/20170126

Disposición No 4362/2014. (2014). Procedimiento para la Resolución de casos sobre sospecha fundada en la Subcategoría Turista. Dirección Nacional de Migraciones, 4 de noviembre de 2014. Buenos Aires, Argentina.

Resolución 618/2014. (2014). Sistemas de Información Anticipada de Pasajeros (API) y Registro de Nombres de Pasajeros (PNR) en los aeropuertos desde 2014, 8 de septiembre de 2014. Buenos Aires, Argentina. Recuperado de http://servicios. infoleg.gob.ar/infolegInternet/anexos/235000-239999/235497/norma.htm

\section{Entrevistas}

Fátima. (10 de abril de 2018). Comunicación personal. Córdoba, Argentina. Joseph. (17 de noviembre de 2017). Comunicación personal. Rosario, Argentina. Lyne. (15 de diciembre de 2017). Comunicación personal. Rosario, Argentina. Michele. (14 de marzo de 2018). Comunicación personal. Córdoba, Argentina. Moise. (6 de abril de 2018). Comunicación personal. Córdoba, Argentina. Robert. (16 de noviembre de 2017). Comunicación personal. Rosario, Argentina. 
Wilguens, Michele, Stéphane (7 de marzo de 2018). Comunicación personal. Córdoba, Argentina.

Carina Trabalón

Argentina. Licenciada en Sociología por la Universidad Nacional de Villa María (UNVm). Doctoranda en Estudios Sociales de América Latina en el Centro de Estudios Avanzados (CEA) de la Universidad Nacional de Córdoba (UNC). Becaria interna doctoral del Consejo Nacional de Investigaciones Científicas y Técnicas (CONICET-Unvm). Líneas de investigación: prácticas estatales de control, regímenes de migraciones y fronteras, migración haitiana y trayectorias. Publicación reciente: Trabalón, C. (2018). Política de visado y regulación de las fronteras. Un análisis desde la movilidad de haitianos en Sudamérica. Polis (Santiago). Revista Latinoamericana, 17(51), 163-186. 